\title{
Hacia la construcción de pedagogías y memorias plurales: explorando aprendizajes en una zona veredal de transición y normalización (Guaviare, Colombia)
}

Towards the Construction of Pedagogies and Plural Memories: Exploring Learnings in a Transitional and Normalization Area (Guaviare, Colombia)

\author{
Deissy Perilla* \\ McGill University, Canadá
}

DOI: $10.22380 / 2539472 X .1046$

\begin{abstract}
RESUMEN
Este artículo presenta un material etnográfico y pedagógico inédito que contribuye - e interpela - a la mirada oficial sobre la pedagogía para la paz, uno de los ejes de los acuerdos de paz en Colombia. Mediante talleres pedagógicos realizados en una zona veredal de transición y normalización (ZVTN) en el Guaviare, con un grupo de 285 excombatientes de las Fuerzas Armadas Revolucionarias de Colombia (FARC) en tránsito hacia la vida civil, se identificaron experiencias locales de aprendizaje que muestran un repertorio de conocimientos adquiridos durante la trayectoria en la guerrilla y en la transición posterior a la firma de los acuerdos. Con base en el análisis de las cartillas trabajadas con el grupo de excombatientes, argumento que urge ampliar la consideración de una pedagogía para la paz incluyendo una pedagogía desde la paz.
\end{abstract}

Palabras clave: memorias, proceso de paz, conocimiento local, pedagogía desde la paz.

\begin{abstract}
$\overline{\text { ABSTRACT }}$
This article presents original ethnographic and pedagogical material that contributes - and questions - the official view of pedagogy for peace, an important dimension of the Peace Agreements in Colombia. Through a series of pedagogical workshops conducted in a transitional and normalization zone (ZVTN) in Guaviare with a group of 285 ex-combatants of the Revolutionary Armed Forces of Colombia (FARC) in transit to civilian life, local learning experiences were identified. These experiences reveal a repertoire of knowledge acquired during their permanence in the guerrillas and in FARC's civil transition after signing the peace agreements. Based on the analysis of the working booklets filled in with this group of ex combatants, I argue that it is urgent to broaden the consideration of a pedagogy for peace through the inclusion of a pedagogy from peace.
\end{abstract}

Keywords: memories, peace process, local knowledge, pedagogy from peace.

Esta obra está bajo licencia internacional Creative Commons Reconocimiento-NoComercialSinObrasDerivadas 4.0

** Magíster en Antropología de la Universidad de los Andes y candidata a doctora en Antropología de la Universidade Federal do Rio Grande do Sul (UFRGS), Brasil. deissy.perilla@gmail.com / https://orcid.org/0000-0002-1457-7113 


\section{Introducción}

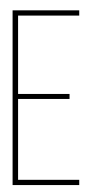

115 de mayo del 2017 un grupo de jóvenes —algunos, miembros de la Veeduría Ciudadana Campamento por la $\mathrm{Paz}^{1}$ — entramos a la zona veredal de transición y normalización (ZVTN) ${ }^{2}$ Jaime Pardo Leal ubicada en el departamento del Guaviare, en la Orinoquía colombiana.

Fuimos invitados por algunas personas de la exguerrilla Fuerzas Armadas Revolucionarias de Colombia-Ejército del Pueblo (FARC-EP) ${ }^{3}$, a quienes conocimos en el evento Vigilias por la Paz $^{4}$. Esta visita ${ }^{5}$ tuvo como fin apoyar el proceso de reincorporación de exmiembros de las FARC-EP a la sociedad civil, a partir de una necesidad reconocida en la vigilia: identificar procesos educativos dentro de esa guerrilla y resaltar el valor que estos tienen para su reincorporación a la vida civil en el marco del Acuerdo Final para la Terminación del Conflicto y la Construcción de una Paz Estable y Duradera, firmado con el Gobierno nacional en el 2016.

Este artículo presenta un conjunto de memorias registradas por medio de una herramienta escrita, denominada cartilla pedagógica, un material etnográfico y pedagógico relevante para producir conocimiento sobre cómo pensarnos dentro de una sociedad en tránsito hacia la paz. Argumento que explorar

1 La Veeduría Ciudadana Campamento por la Paz está compuesta por un grupo heterogéneo de 37 ciudadanos colombianos que, después de participar en la protesta Campamento por la Paz (en octubre y noviembre del 2016), decidieron continuar su participación política, entendida como una participación ciudadana activa, independiente de los marcos oficiales de representación del Estado (Congreso), para desarrollar una agenda de monitoreo de algunos puntos de la implementación de los acuerdos de paz. Entre sus actividades se encuentran: seguimiento a debates en el Congreso, elaboración de informes, publicación de comunicados, visitas a las ZVTN. La Veeduría está amparada por el numeral 2.2.5 de los acuerdos sobre “Control y veeduría ciudadana" (Veeduría Ciudadana Campamento por la Paz 2016).

La Oficina del Alto Comisionado para la Paz caracteriza las ZVTN como “áreas temporales y transitorias, definidas, delimitadas y concertadas entre el Gobierno nacional y las FARC-EP para llevar a cabo el proceso de dejación de armas e iniciar el tránsito hacia la legalidad de las FARC-EP". Estas zonas entraron en vigencia en diciembre del 2016 y debían durar hasta el 31 de mayo del 2017 (Oficina del Alto Comisionado para la Paz 2017, 3).

3 La situación actual de asesinatos de líderes sociales y excombatientes, desde la firma de los acuerdos de paz, me obliga a mantener en anonimato los nombres de excombatientes, líderes comunitarios y miembros de la mencionada Veeduría que hicieron parte de esta experiencia.

$4 \quad$ Este evento de carácter humanitario fue organizado por la Fundación de Derechos Humanos del Oriente Colombiano (DHOC) y por algunos miembros de la comunidad ecuménica colombiana.

5 En conversaciones previas entre miembros de la Veeduría con funcionarios de la Oficina del Alto Comisionado para la Paz se nos aclaró que las visitas de civiles a estas zonas eran permitidas, siempre y cuando se llevaran a cabo en unos "anillos" o zonas especiales, tal como sucedió en nuestras actividades. 
procesos educativos y de memoria entre grupos que han participado y padecido la guerra en Colombia puede dar luces para aprender a mirarnos desde adentro, es decir, desde los diferentes territorios, desde la guerra, desde las memorias y los procesos de aprendizaje poco conocidos o ignorados por las narrativas hegemónicas. El objetivo de este trabajo involucra, por lo tanto, una tensión metodológica que merece ser resaltada desde el inicio y que se refiere a la tendencia a considerar a las FARC-EP de modo directo y unánime como enemigo de la nación. Esta tensión acompaña, por lo general, a quienes desde la investigación recopilamos y analizamos este tipo de narrativas para, en muchos casos, exponerlas en espacios académicos y no académicos. Por ello, escribir sobre esta guerrilla, aún en plena transición hacia la paz, suele implicar señalamientos y eso condiciona nuestro ejercicio. No obstante, es importante promover el debate sobre temas como la memoria y la pedagogía en escenarios propios del posacuerdo.

Al escribir estas páginas no paso por alto la historia de violencia desencadenada por la guerrilla en los últimos cincuenta años en el territorio colombiano. No pretendo exponer aquí una mirada romantizada e idealizada de las FARC-EP; mucho menos, una mirada estigmatizada o deshumanizante. La motivación principal para presentar parte de un material inédito se enmarca en el momento histórico de la compleja transición hacia la paz en que se encuentra el país, donde la narrativa de la guerra entre "bandos" definidos como buenos o malos precisa urgentemente de una revisión desde las ciencias sociales para avanzar hacia la construcción de paz. El aporte metodológico y analítico presentado en este artículo se basa en el resultado de un material pedagógico trabajado y construido con algunos excombatientes, a partir de los conocimientos afianzados durante su trayectoria guerrillera y en los momentos posteriores a la firma de los acuerdos de paz.

Estos movimientos hacia la paz requieren varias transformaciones sociales, entre ellas, una propuesta educativa que recupere saberes locales, entendidos como aquellas prácticas culturales concretas orientadas a producir conocimiento sobre las experiencias vividas, generadas o reelaboradas durante el conflicto. Comprende también memorias desde lo rural y desde las regiones en relación con personas y grupos que han vivido la guerra, como es el caso de las FARC-EP. Por lo tanto, en este escrito la educación será entendida, desde esos saberes locales, como un trabajo sobre la cultura (Ávila 1994, 36) y como "una práctica social, forma de acción, manera de actuar, roles que ponemos en escena, papeles que nos distinguen de los otros actores, papeles que tienen que ver con la génesis y la construcción de nuestra identidad y memoria social” (37). Asimismo, la pedagogía aquí se entiende como "la disciplina que conceptualiza, aplica y 
experimenta los conocimientos referentes a la enseñanza de los saberes específicos en las diferentes culturas" (Zuluaga et al. 1988, 24). En otras palabras, la pedagogía no es solo un medio o instrumento - como las llamadas ciencias de la educación lo han querido entender ${ }^{6}$-, sino una disciplina que crea y recrea contenidos específicos de cada cultura, sociedad o comunidad. En este sentido, desempeña un papel importante en las maneras diversas de conocer, vivir, identificarse, recordar y olvidar.

Ahora bien, ¿cómo se vinculan estas definiciones provisionales de educación y pedagogía con la memoria dentro de la construcción de paz en el llamado posconflicto? La memoria es un mecanismo cultural clave en procesos de reconstrucción de tejido social, dignificación, rehumanización y "empoderamiento de las víctimas, sus formas de resistencia frente a la agresión, a la violencia, y a la transformación de los imaginarios que perpetúan la violencia” (Villa 2009, 73). También es crucial en tiempos de construcción de paz después de largos periodos de violencias, pues tales transformaciones implican, entre muchas cosas, reinterpretar las experiencias vividas, cuestionar la propia identidad, reorientar la forma de recordar (u olvidar) el pasado hacia escenarios políticos emergentes. Ampliaré algunos de estos puntos mediante hallazgos que resultaron del ejercicio de elaboración y diligenciamiento de las cartillas pedagógicas con miembros de esta organización ${ }^{7}$.

\section{Hacia una pedagogía desde la paz}

Un movimiento que propongo en este artículo es el paso de una pedagogía para la paz a una pedagogía desde la paz, en el marco de los múltiples esfuerzos metodológicos para la construcción colectiva de procesos educativos en tiempos de reconstrucción; algunos de estos esfuerzos surgen de proyectos del Programa Nacional de Educación para la Paz: Educapaz ${ }^{8}$. Por pedagogía para la paz entiendo la mirada institucional sobre educación y paz expuesta en el Acuerdo Final para la Terminación del Conflicto y la Construcción de una Paz Estable y

$6 \quad$ Para ampliar el debate, véase Zuluaga et al. (1988, 4-9).

7 Uso la palabra organización como una categoría émica de autoidentificación, es decir, comúnmente utilizada por los miembros de la exguerrilla.

$8 \quad$ Para ampliar, véase “Sembrando oportunidades. Informe de gestión 2016-2017" (2017), disponible en https://educapaz.co/leenos/informes-de-gestion/ 
Duradera. Mi argumento central es que esta propuesta precisa entrar en diálogo con y alimentarse de una pedagogía desde la paz; es decir, desde las memorias sobre los saberes y procesos educativos locales producidos en la guerra. Lo anterior debe buscarse desde el trabajo junto con las comunidades afectadas por el conflicto armado en Colombia, en sus propios territorios, indagando profundamente por sus propias historias y memorias.

Desglosemos lo anterior. Como se puede observar en el acuerdo de paz vigente firmado en el 2016, la mención a la educación para la paz es básica y tímida, no obstante, importante e histórica. El acuerdo habla de una "pedagogía para la paz" en el punto sobre la reforma rural integral (apartado 1.3.2.2). Se enfoca en la "educación rural", que implica el acercamiento de las instituciones académicas a las comunidades rurales, con el fin de acabar con el analfabetismo, especialmente en la primera infancia, y promover la producción en el campo. El acuerdo propone lograrlo por medio de la difusión de ofertas técnicas, tecnológicas y universitarias, así como a través de "modelos flexibles de educación”, mejoramiento de infraestructuras en las escuelas, garantía de gratuidad, disponibilidad de becas, entre otros mecanismos. Por otro lado, se hace mención de una "pedagogía para la paz" en el punto 3, sobre el fin del conflicto (numeral 3.2.2.6), que señala la importancia de "una identificación de necesidades del proceso de reincorporación”, así como hacia proyectos comunes e individuales de los excombatientes. Finalmente, el numeral 3.2.2.7, que versa sobre las "garantías para una reincorporación económica y social sostenible”, propone una pedagogía para la paz a partir de tres voceros de cada ZVTN cuyo encargo es hacer pedagogía de los acuerdos en sus municipios correspondientes.

El acuerdo cuenta con la virtud de mencionar la importancia de la educación para construir la paz, pero no explica en qué consiste esa importancia y cómo se entiende la educación en un contexto de posconflicto. Propone, de entrada, implementar "procesos educativos formales" (en academias, institutos técnicos, tecnológicos, etc.), es decir, entendiendo la educación como algo que ocurre mayoritariamente en ciudades y dentro de los salones de clase. En tal sentido, y en el marco de la apuesta de una pedagogía desde la paz, se requiere posicionar la necesidad de propuestas de educación que se alimenten de otros lugares, procesos y contextos. Reconocer los procesos educativos como locales y diversos implica ampliar el foco hacia las múltiples versiones de la educación como experiencia vivida.

Sugiero que una propuesta de educación para una sociedad que se encuentra en tránsito hacia la paz debe partir de reconocer y defender el derecho que cualquier individuo tiene - sin importar a qué grupo pertenezca-de intervenir 
activamente en la construcción de su memoria y de su futuro, así como en la transformación de sus condiciones de existencia. Las pedagogías en su diversidad son también formas de resistencia y pueden surgir desde la oposición a un gobierno o en procesos sociales como la insurgencia, el cimarronaje, la afirmación, la reexistencia, la rehumanización, entre otros (Walsh 2013).

En Colombia, la emergencia de la memoria como un campo de interés de la antropología, en el marco de la implementación de los acuerdos de paz, está ligada a su conceptualización como una herramienta política mediante la cual las versiones de la historia que emergen durante un proceso de paz son valiosas en tanto sean diversas e incluyentes. Como argumenta Elizabeth Jelin (2002), la cultura de la memoria de las últimas décadas, en la que los testimonios son centrales, está unida a las disputas políticas de diferentes actores sociales por hacer visibles y lograr el reconocimiento de los sentidos que estas comunidades construyen por sí mismas (56).

Hacer pedagogía desde la paz implica un esfuerzo por comprender las memorias locales, por ejemplo, las contribuciones de la historia oral a las ciencias sociales y humanas (Bastide 1983; De Queiroz 1988; Fernandes y Gattas 1971) que le han dado un lugar legítimo al análisis de las memorias de los excluidos. Como lo explica Michael Pollak (1989): "la historia oral resaltó la importancia de las memorias subterráneas que, como parte integrante de las culturas minoritarias y dominadas, se oponen a la 'memoria oficial' para el caso de la memoria nacional” (4). En el asunto que nos ocupa, esta afirmación supone actuar y construir conocimiento junto con los otros, a partir de sus propias necesidades y cuestionamientos; escuchando, accediendo a conocimientos internos y dándoles valor, con la expectativa de ponerlos a circular en escenarios y públicos más amplios en aras de la construcción de paz.

Las memorias, narrativas y conocimientos recogidos en las cartillas pedagógicas permiten inferir que los miembros de la exguerrilla han tenido experiencias que van mucho más allá del entrenamiento militar, que dan cuenta de procesos educativos complejos y diversos. En este sentido, es urgente dar a conocer públicamente cómo los excombatientes imaginaron sus transiciones a la "vida civil” aludiendo a sus memorias y a procesos educativos en el contexto de un momento tan único como fue el traslado a las ZVTN. A lo largo de este escrito sugeriré la importancia de la construcción colectiva de un modelo de educación desde la paz en coherencia con las necesidades locales, que a su vez dialogue e interpele la visión de pedagogía para la paz presente en los acuerdos de La Habana. 


\section{Las zonas veredales de transición y normalización (ZVTN): un campo etnográfico emergente}

Desde su creación en el 2017, las ZVTN han sido consideradas lugares de producción de conocimiento sobre la paz y espacios para formular preguntas importantes para la antropología colombiana. Al respecto, se destacan los trabajos de Juan Felipe Hoyos (2017), en la Zona de La Paya en Tumaco (Nariño); las investigaciones de Alhena Caicedo y Carlos Alberto Benavides (2017), en la Zona de Buenos Aires (Cauca), sobre el papel de la etnografía en estas transiciones, las nuevas dinámicas en los territorios campesinos y el papel de la memoria en estos escenarios; así como el trabajo de Vianney García y Libia Tattay (2017) en ZVTN cercanas a los territorios del pueblo nasa, en el que retoman la problemática de la compleja relación histórica de las FARC-EP con varias comunidades. Está también el trabajo de Rodrigo Valero (2017) en la ZVTN El Oso, en Planadas (Tolima), quien se pregunta si la institucionalidad, dado el nuevo orden del Estado, estaría dispuesta a dialogar con otros "órdenes del mundo" existentes en estos territorios. Este último tema también es discutido por Keren Marín y Nicolás Espinosa (2017), que desde la ZVTN de La Macarena analizan las tensiones que se tornan palmarias ante el sistemático asesinato de líderes sociales en esta región.

Varios colectivos de estudiantes, fundaciones de carácter religioso, colectivos de paz, entre otros grupos, se movilizaron a estas zonas con varios objetivos. Gran parte buscó respaldar el desplazamiento de las FARC hacia las 19 zonas veredales de transición y normalización, y los siete puntos transitorios de normalización (PTN) ${ }^{9}$. El proceso de reagrupamiento en estos espacios inició oficialmente el 28 de enero del 2017. Este paso fue esencial después de que el acuerdo de paz fuera refrendado en el Congreso de la República, lo que dio inicio al día $D$, a partir del cual se contarían 180 días para el cese al fuego bilateral definitivo, la dejación de armas por parte de la guerrilla y el largo proceso de reincorporación de la población exguerrillera a la vida civil.

$9 \quad$ La Oficina del Alto Comisionado para la Paz define las ZVTN y los PTN como "áreas temporales y transitorias, definidas, delimitadas y concertadas entre el Gobierno nacional y las FARC-EP para llevar a cabo el proceso de dejación de armas e iniciar el tránsito hacia la legalidad de las FARC-EP”. Consultado el 27 de septiembre del 2019 en http://www.altocomisionadoparalapaz. gov.co/procesos-y-conversaciones/Documentos\%20compartidos/ABC-Zonas-veredales.pdf 
En diciembre del 2017 las ZVTN pasaron a ser espacios territoriales de capacitación y reincorporación ${ }^{10}$. Estas zonas, cuya fecha límite de ocupación debía ser mayo del 2017, fueron habitadas hasta mediados de agosto de ese mismo año y se convirtieron en espacios permanentes de vivienda "para la capacitación y la reincorporación”. Según cuentan los mismos exguerrilleros en conversaciones sostenidas durante nuestras visitas, la extensión del plazo se explica por los repetidos incumplimientos del Gobierno nacional para garantizar zonas habitables y debidamente equipadas: retraso en la llegada de materiales adecuados para el abastecimiento de las viviendas, implementos de salud y alimentos, y en la construcción y dotación de espacios de capacitación y educación necesarios para transitar a la vida civil.

Por su parte, para las personas de la exguerrilla que habitan en la región del Guaviare es evidente que una razón de peso para la permanencia en las ZVTN tiene que ver con el miedo creciente de mudarse a ciudades y otras regiones, debido a que se considera como una región con una base social de la guerrilla mucho más consolidada en medio de una fuerte e histórica pugna con otros grupos armados. Este temor también se origina en el estigma al que tendrían que enfrentarse fuera de territorios considerados “propios” y a la percepción de los excombatientes como personas no aptas para convivir en una sociedad no militarizada ${ }^{11}$.

\section{Talleres pedagógicos en la ZVTN Jaime Pardo Leal}

Como se mencionó, los talleres realizados con este grupo de excombatientes en la zona veredal Jaime Pardo Leal tuvieron como objetivo crear unos espacios de identificación, reconocimiento y valorización de procesos de aprendizaje locales, así como de escuelas de formación que funcionaban intrafilas. Esas escuelas fueron espacios de aprendizaje surgidos en medio de la guerra y que, si bien incluían la formación militar, también integraban conocimientos sobre pedagogía, formación política, salud, medio ambiente, arte, comunicación, entre otros campos de saber.

\footnotetext{
10 Conforme a lo decidido por la Comisión de Seguimiento, Impulso y Verificación a la Implementación del Acuerdo Final (CSIVI), Decreto 1274 del 2017 y Decreto 2026 del 2017.

11 Esta conclusión se extrae de conversaciones informales sostenidas en nuestras visitas con un grupo de aproximadamente treinta personas.
} 
Es importante aclarar que las escuelas de formación no fueron descritas en profundidad por nuestros interlocutores, puesto que no se nos permitió llevar a cabo entrevistas formales y contamos con un tiempo bastante limitado para desarrollar los talleres con las cartillas. En los talleres participaron 285 personas $^{12}$ que compartieron con nosotros recuerdos, dibujos, textos y mensajes en las cartillas trabajadas. Esta actividad fue concebida en colaboración con alias Alejandra y contó con la aprobación de Mauricio Jaramillo (Jaime Parra, actual miembro del partido político FARC $)^{13}$.

A la ZVTN Jaime Pardo Leal, localizada en el departamento del Guaviare (figura 1), se trasladaron aproximadamente 500 exmiembros de las FARC-EP durante enero del año 2017, la mayoría provenientes de la zona de agrupamiento previa a esta fase en los llanos del Yarí (departamento del Meta). El traslado se realizó por medio de caravanas terrestres en las que hubo mucha participación y apoyo por parte de ciudadanos provenientes de varios pueblos aledaños, así como de Bogotá y otras ciudades. El proceso de traslado a esta ZVTN duró casi un mes. Para el momento de nuestra visita, la mudanza ya había concluido, aunque el grupo continuaba esperando los recursos que prometió el Gobierno para garantizar una apropiada instalación, alimentación y abastecimiento, así como la prestación de servicios especiales para mujeres embarazadas.

Agradezco a Jaime Alberto Parra Rodríguez, también conocido como Mauricio Jaramillo o "el Médico", por su disposición para responder nuestras inquietudes, y por habernos abierto un espacio de diálogo sobre pedagogía y paz. Jaime Parra estudió Medicina en la Universidad Nacional, hizo una especialización en dermatología en la Unión Soviética y fue el encargado de crear la Escuela de Medicina dentro de las FARC-EP (conversación personal). Vale la pena aclarar que Jaime Parra aprobó la aparición de su nombre en este artículo, por tratarse de una figura pública en reincorporación a la vida civil y política. También quiero agradecer a alias Alejandra, exmilitante de las FARC-EP, cuyo rol como educadora, poeta, escritora y artista en el momento de la firma de los acuerdos fue clave para la transición de los excombatientes a la vida civil, así como para nuestro encuentro al generar puentes de diálogo sobre temas de educación y pedagogía. tariado de las FARC-EP. Durante el ejercicio realizado en la ZVTN, era nombrado como "el camarada Mauricio". No fue la única persona presente en los talleres, pero sí la única que se puede mencionar en este escrito por los acuerdos de confidencialidad. 
Figura 1. Mapa de las ZVTN

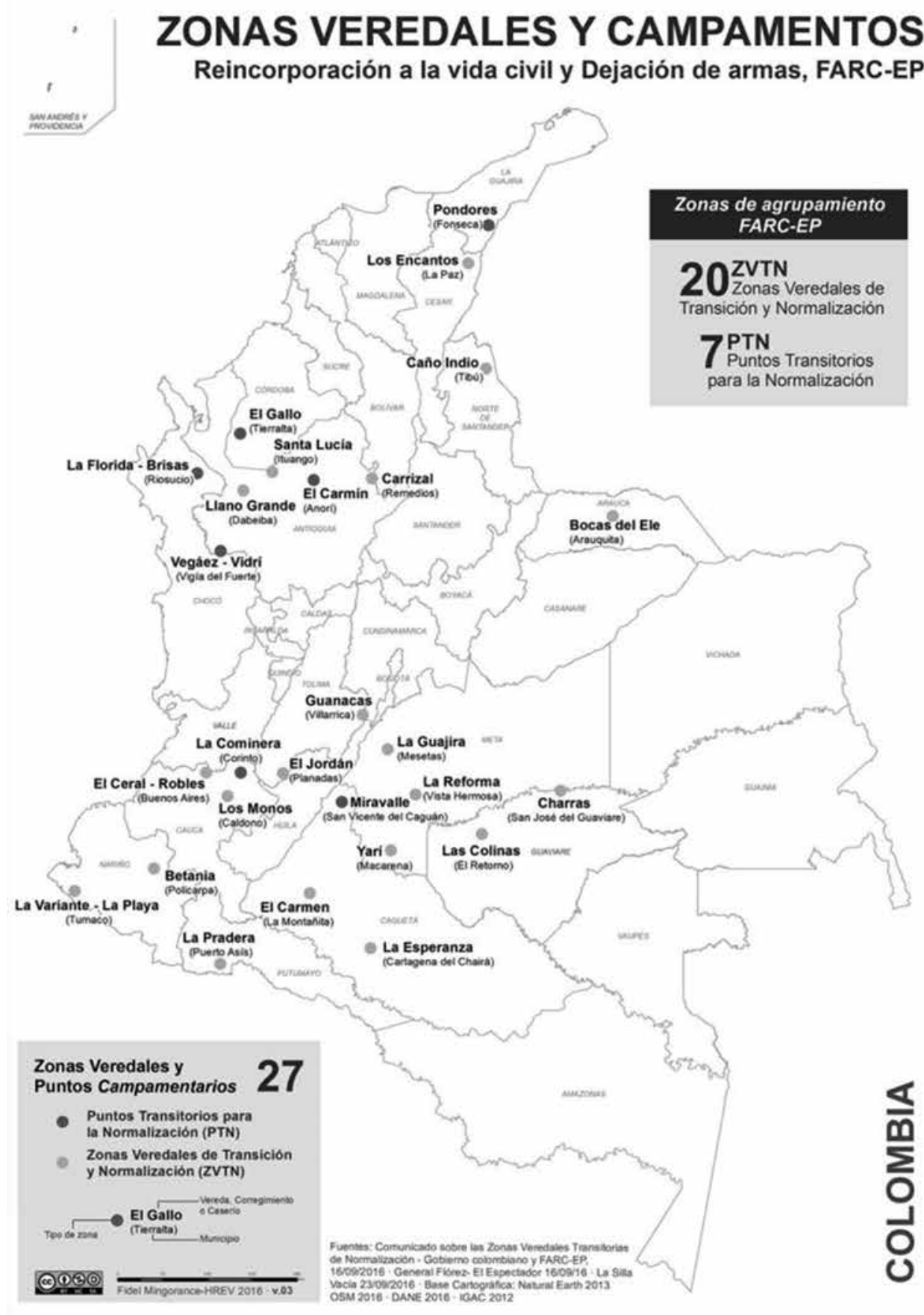

Fuente: Oficina del Alto Comisionado para la Paz (2017b, 2). 
Al llegar observamos una población organizada en grupos de trabajo para la transición: un grupo formado por los encargados de la construcción de las viviendas provisionales, un comité de bienvenida para la población civil que llegara desde las diferentes regiones, uno encargado de "ranchar" (como se les llama a las labores relacionadas con la preparación de alimentos dentro de la guerrilla), un grupo responsable de la seguridad y vigilancia, otro encargado del transporte para el ingreso y egreso de visitantes, y el grupo central de líderes y coordinadores del lugar, entre ellos alias Mauricio Jaramillo, alias Iván Ali y alias Fidel, quienes nos recibieron en el cambuche central para evaluar nuestra propuesta y conversar sobre lo que ha sido su proceso de mudanza a la ZVTN.

Para la realización de los talleres nos encontramos con un grupo en constante y diario aprendizaje de lo que significa el tránsito hacia la "vida civil". Esto quiere decir que, además de observar estructuras típicas de "la guerrillerada” (formación en escuadrones, distribución jerárquica de zonas, habitación en cambuches, sectores de vigilancia, ranchos, espacios de formación política), nos encontramos con la zona de transición como tal, es decir, con las nuevas bibliotecas, las áreas de recreación y deporte, una panadería que había resultado de las clases dictadas por el Servicio Nacional de Aprendizaje (SENA), construcciones de viviendas fijas, entre otros.

\section{Los talleres, las cartillas y las memorias}

Durante el trabajo realizado con la Veeduría Ciudadana en la zona, muchos excombatientes manifestaron tener como meta terminar sus estudios básicos de bachillerato e ingresar a instituciones de educación superior para realizar estudios de pregrado universitario en diferentes áreas del conocimiento: medicina, antropología, diseño, ingeniería, educación, entre otras. Con relación a tales objetivos, se pudo evidenciar que el punto que ha generado mayores dudas, preguntas e incertidumbres tiene que ver con la prueba Saber del Instituto Colombiano para la Evaluación de la Educación (Icfes). Este examen estandarizado fue creado por el Ministerio de Educación para “evaluar el nivel de la educación media en el país, así como para proporcionar información a la comunidad educativa sobre el desarrollo de las competencias básicas que debe desarrollar un estudiante durante su paso por la vida escolar” (“Glosario Icfes” 2019). El debate sobre esta instancia de evaluación incluyó desde el desconcierto inicial de quienes no conocían su existencia hasta la consideración de las dificultades que la prueba podría 
conllevar para los excombatientes. Nos propusimos entonces realizar unos talleres pedagógicos, cuyo objetivo era identificar conocimientos, habilidades, saberes y experiencias educativas durante sus trayectorias de vida en la guerrilla.

Según un censo interno hecho por las FARC en el 2017 sobre el nivel de escolaridad (previo al ingreso a la guerrilla) en esta ZVTN, de las 466 personas registradas, el $1 \%$ es analfabeta, el $5 \%$ hizo primer grado de primaria, el $14 \%$ hasta segundo y el 11\% hasta tercer grado. El $6 \%$ llegó al cuarto grado y el 17\% a quinto. En el nivel de bachillerato, el 11 \% cursó hasta el grado sexto y el 10\% hasta séptimo. Quienes cursaron hasta el grado once representan menos del $7 \%$ de esta población. En cuanto a la educación universitaria, solo una persona se graduó de la carrera de medicina (alias Mauricio Jaramillo), y otras siete cursaron algunos semestres en bacteriología, español, medicina, antropología, filosofía, ingeniería química y economía. Un $9 \%$ de las personas que no fueron a la escuela aprendieron a leer y a escribir dentro de la guerrilla.

Con este sondeo en mente diseñamos una cartilla pedagógica como instrumento experimental para acceder a esos universos pedagógicos poco o nada conocidos por nosotros. Nuestra idea era construir un escenario en el que estas experiencias de vida y procesos de conocimiento previos a la fase del posconflicto entraran en diálogo con las diferentes miradas que de este lado tenemos sobre la construcción de paz en escenarios educativos colectivos. Para consolidar la herramienta fue necesario, en primer lugar, un diálogo permanente con alias Alejandra, quien ha trabajado durante décadas en procesos de formación y educación desde diversas áreas (artes, escritura, comprensión de lectura y pedagogía), en el contexto del conflicto armado y bajo las condiciones de ilegalidad de la vida guerrillera.

La cartilla surgió para explorar una ruta que conecte los dos mundos involucrados en el tránsito de los habitantes de la ZVTN: el de la vida civil y el de la vida no civil; nació con una hoja en blanco que alias Alejandra y yo empezamos a llenar poco a poco con algunas preguntas: ¿cómo podemos saber qué es lo que ustedes saben? (pregunta mía) o ¿cómo tornar valioso lo que aprendimos en la guerra para no empezar desde cero? (pregunta de ella). Con estos interrogantes en mente, Alejandra (que participaba a la distancia y mediante comunicaciones telefónicas), los estudiantes de antropología David y Valentina, el pedagogo Alejandro y yo conformamos un equipo que estableció un conjunto de puntos y preguntas en un documento didáctico, fácil de trabajar y cuyo contenido permitiera crear un puente entre diferentes visiones de educar y ser educado, teniendo en cuenta que la educación es una práctica histórica y sociocultural (Ávila 1994), 
ligada a la memoria y a los procesos locales, donde las pedagogías y saberes propios son fundamentales.

Los talleres se realizaron con la presencia de algunas autoridades de la entonces organización en tránsito FARC-EP (como alias Mauricio Jaramillo), quienes convocaron a 285 hombres y mujeres excombatientes que tuvieran entre 17 y 60 años. Durante cuatro días se llevaron a cabo cinco sesiones con la participación de aproximadamente 60 personas cada una. Los espacios fueron coordinados por nosotros, un equipo de cuatro personas, y cada taller duró aproximadamente dos horas. Ese tiempo fue distribuido de la siguiente manera: 1) una introducción de la propuesta del taller y una charla sobre las pruebas Saber del Icfes. Esta charla consistió en explicar a grandes rasgos qué eran estas pruebas, cómo se aplicaban, la importancia de hacerlas y las estrategias para tomar el examen de la forma más práctica y en el menor tiempo posible. 2) Entrega de un simulacro de las pruebas Saber para ser resueltas no en el taller sino en sus tiempos libres, pero con nuestro apoyo durante la estadía de cinco días en esta ZVTN. 3) El trabajo con las cartillas pedagógicas diseñadas para conocer sus aprendizajes internos, así como sus memorias del conflicto armado. Esta fue la parte más larga de la actividad, a la que se le dedicó aproximadamente una hora.

Las cartillas contenían nueve puntos, entre preguntas y ejercicios para ser resueltos de forma individual o colectiva ${ }^{14}$, con respuestas escritas o mediante dibujos o ilustraciones ${ }^{15}$. Las cartillas de seis páginas debían ser trabajadas en una hora (figura 2).

El nivel de lectura y escritura en la comunidad es alto; solo el 5\% no sabía escribir. Con estas personas se realizó un acompañamiento y apoyo especial, con la colaboración del resto de participantes de los talleres, así como de nosotros, los coordinadores de estos espacios. básicos de comprensión de textos y de escritura, algunos no están tan familiarizados con estas actividades. Vale aclarar que se cuenta con la autorización para mostrar estos dibujos de forma pública, respetando la voluntad de anonimato de sus autores. 
Figura 2. Modelo de la Cartilla pedagógica

"Veeduria Campamento por la Paz" con Registro Público PDCPL 21-481 a Nivel Territorio Nacional

Taller de construcción y exploración de experiencias educativas en la ZVTN "Jaime Pardo Leal" (Guaviare)

\section{CARTILLA I}

\section{Vamos a dialogar sobre educación ¿nos le medimos?}

1. Les proponemos una lectura para que reflexionemos sobre las preguntas que se encuentran al final.

\footnotetext{
* Entiéndase por Occidente la forma de pensar y actuar en lo político, económico y cultural tanto de Europa como de Estados Unidos
}

\section{SOBRE LA EDUCACIÓN DE OCCIDENTE}

-¿Y qué opinaba ela?

-Que cuando tienes claro lo que quieres no te planteas si estás errando el camino.

-En Occidente no se os educa en el sacrificio, ni en la paciencia, ni en la satisfacción de lo bien hecho. No os enseñan que la única vía para desarrolar una vida plena es tener una meta clara; pero no alcanzarla, sino para tender hacia ela. No os dais cuenta de que lo más satisfactorio es ser consecuentes con nuestros actos. Tú careces de esa meta, y por eso te lanzas sin pensar hacia todo lo que se te pone por delante. Y ello te lleva a caer en el desorden, en el ruido, culpando al que tienes más cerca de tus propias limitaciones. Si tuvieras ese objetivo vital claro, como te decia Asha, estarias convencido en todo momento de estar haciendo lo correcto. Y, lo más importante, te sentirias libre, que es algo imprescindible para realizamos en todas las esferas, para ser sinceros y dejar que los que tenemos a nuestro alrededor nos ayuden a mejorar.

-Es duro lo que me dices.

-No te estoy culpando. Sólo quiero que sepas que si no legas a comprender estas verdades básicas todo lo que has pasado no te habrá servido de nada. (...)

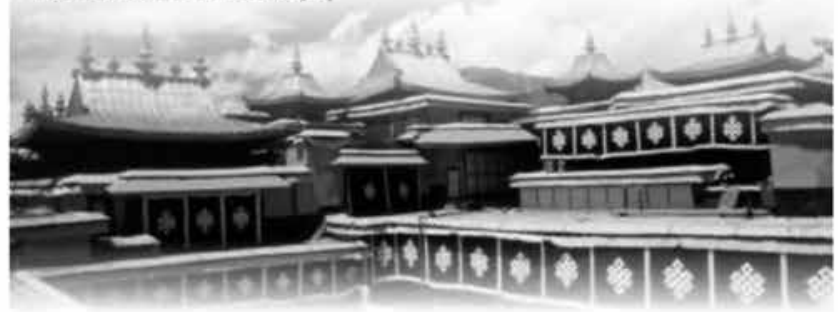

Tomado de: https://conlaspalabras.wordpress.com/curso-0708/mas-alla-del-aula/

VOL. $56, N^{0} 1$ 
"Veeduría Campamento por la Paz" con Registro Público PDCPL 21-481 a Nivel Territorio Nacional

Taller de construcción y exploración de experiencias educativas en la ZVTN "Jaime Pardo Leal" (Guaviare)

- ¿Cuál es la importancia o el propósito de educarse?

- ¿Qué herramientas brinda la educación en la vida de las personas?

- ¿Por qué es importante la educación en un escenario de post-conflicto?

2. Cuéntenos qué opiniones, pensamientos, recuerdos o emociones les genera el siguiente poema:

\title{
REVOLUCIÓN
}

\author{
Los que no ven, nos dicen ciegos, \\ pero tú nos has enseñado a ver el color \\ del tiempo que viene. \\ Los que no oyen, nos dicen sordos, \\ pero tú nos has enseñado a escuchar en todas \\ partes el ágil sonido de la ternura humana. \\ Los cobardes nos dicen cobardes, \\ pero contigo nos enfrentamos a las sombras \\ y les cambiamos el rostro. \\ Los criminales nos dicen criminales, \\ pero contigo revivimos la esperanza \\ le marcamos el alto al crimen, a la \\ prostitución, al hambre. \\ Y le ponemos ojos, oídos, alma \\ al corazón del hombre. \\ Los racistas nos dicen antihumanos \\ pero contigo le damos al odio \\ su tumba mundial en la ciudad de los abrazos. \\ Nos dicen tantas cosas. \\ Y los que las pronuncian \\ olvidan -estúpidos que son- \\ que sus nietos amarán mañana jubilosamente \\ la palabra estrellada de tu nombre, \\ Revolución. \\ Tomado de: https://espaciolibremexico.wordpress.com/2012/04/14/los-versos-de-la-juventud-rebelde-zapatista/
}

3. A lo largo de nuestra vida han existido personas que han marcado nuestros caminos de diferentes maneras ¿A usted qué persona le produce admiración y por qué? ¿Cómo ha influenciado su vida?

\section{Échenos un cuento}

4. La vida se compone de varias etapas y ciclos, en los cuales aprendemos en diferentes espacios y con muchas personas. Por ejemplo, ¿Ustedes tienen recuerdos del lugar donde 
"Veeduria Campamento por la Paz" con Registro Público PDCPL 21-481 a Nivel Territorio Nacional

Taller de construcción y exploración de experiencias educativas en la ZVTN "Jaime Pardo Leal” (Guaviare)

estudiaron antes de pertenecer a la organización? si es así, háblenos, escribanos o dibújenos algunos de esos recuerdos.

5. Como hemos visto, la educación es una experiencia vital y todos hemos aprendido de diferentes maneras y en diferentes contextos, entonces ¿Qué importancia tienen para ustedes las escuelas de formación de las FARC-EP? ¿Cómo lo podrían plasmar a través de un dibujo grupal? Puedes elaborar el dibujo con las personas más cercanas a ti.

6. La educación y el aprendizaje se dan en el diario vivir. Con esta idea en mente, ¿Cuál ha sido la principal habilidad o trabajo que ha aprendido dentro de la organización FARC-EP? ¿Cómo ha logrado adquirirla?

7. Sabemos que desde el inicio de los diálogos de paz ustedes han tenido varios lugares de vivienda, esto ha significado muchos cambios y nuevas experiencias ¿Qué ha cambiado desde su traslado a la ZVTN? Narre cómo ha sido esa experiencia desde que salió de los sitios de pre-agrupamiento, hasta su instalación y permanencia en la zona veredal. ¿Qué tal si escribimos un pequeño cuento o hacemos un dibujo?

\section{¿Y el futuro qué?}

8. Teniendo en cuenta que existen diversos procesos educativos dependiendo de las realidades vividas, ¿Considera que la educación recibida en la organización tendrá importancia luego de su tránsito a la vida civil?

9. Queremos conocer sus sueños y anhelos, les proponemos en este punto que plasmen en un dibujo cómo imaginan su futuro en 10 años.

\section{GRACIAS POR COMPARTIRNOS SUS SABERES. LA EDUCACIÓN ES UN TRABAJO EN CONSTRUCCIÓN CON EL OTRO!}

Fuente: fotografía de la autora.

El trabajo por sesión respondió a la dinámica de cada grupo, es decir, algunos grupos estaban más dispuestos a un trabajo colectivo; otros, a un trabajo individual; algunos tenían más disposición para estos talleres mientras que otros, no tanto (especialmente en las sesiones nocturnas). También dependió de la voluntad de cada participante. No obstante, tendió a realizarse en subgrupos de doce a quince personas, aunque el documento debía ser respondido de forma individual. Esto implicó la consulta a otros compañeros o amigos en cada sesión. Cada subgrupo contó con nuestra guía y apoyo; nos encargamos de resolver dudas sobre el sentido de las preguntas, pero principalmente apoyamos el proceso de escritura, esto es, procuramos ayudar a las personas que no se sentían con las habilidades de escritura necesarias para responder cada punto. En estos casos, escribimos lo que los participantes nos dictaban. 
Los talleres finalizaron con una plenaria en la que los participantes tenían la palabra para manifestar su opinión sobre el ejercicio y compartir algunas de las respuestas de las cartillas o algunas impresiones sobre el trabajo realizado. En esta parte de cierre, la participación fue más tímida. Tan solo algunas personas tomaron la vocería, principalmente para agradecer nuestra presencia en la ZVTN, así como la explicación sobre las pruebas Saber. Asimismo, los excombatientes resaltaron la actividad como un espacio de construcción colectiva que buscó darles valor a sus aprendizajes y memorias. Una de las intervenciones mencionó lo importante que fue para ellos ser escuchados, para que la sociedad civil entienda "por qué tomamos el camino de la lucha armada". Desde nuestro equipo de trabajo también hubo algunas reflexiones destacables, como la de Alejandro:

No dejan [los excombatientes] de ser pelaos como uno, que, por diversas razones, y por la vida que les tocó y el lugar del país donde les tocó nacer, pues se vieron obligados a coger un fusil o se los cargaron, o lo que sea, pero siguieron teniendo esos sueños que tiene cualquier pelao que uno entrevista cuando está en once, o sea, “quiero estudiar, quiero ir más allá”. No sé si era por las enseñanzas de Mauricio, que era médico, pero muchos estaban interesados en continuar sus procesos educativos en enfermería o medicina. Y las veces que conversábamos con Mauricio, él siempre mostraba ese interés porque la gente se formara, se capacitara. (Entrevista con Alejandro Cortés, 22 de abril del 2018)

\section{Saberes internos: hacia una caracterización de procesos educativos propios}

Con el objetivo de explorar las memorias de los aprendizajes propios durante la permanencia en la guerrilla y en el proceso de reintegración iniciado en el 2017, me enfocaré en las respuestas dadas a los puntos 1 (sobre la importancia o el sentido de educarse) y 6 (sobre las habilidades o trabajos aprendidos en la organización FARC-EP) de las 285 cartillas diligenciadas. Estos dos puntos generaron respuestas asociadas a los procesos de aprendizaje. A continuación, presentaré algunos resultados y las conclusiones preliminares que aportan al objetivo de este trabajo. En la siguiente tabla se relacionan tres columnas con categorías y subcategorías émicas, donde el número entre paréntesis indica las personas que las mencionaron. La primera columna es el campo de aprendizaje al que se 
refirieron los 285 participantes; la segunda presenta las subcategorías usadas para los contenidos específicos de los procesos de aprendizaje mencionados en la primera columna, y en la última, a modo de ejemplo de cada categoría, se reporta un fragmento de lo dicho por los participantes.

La tabla es una herramienta que posibilita múltiples caminos analíticos; sin embargo, para los objetivos de este trabajo no se requiere un análisis exhaustivo de cada pregunta, sino que bastará con enfocarse en las dos preguntas mencionadas ${ }^{16}$. Vale resaltar que la mayoría de subcampos presentados son aprendizajes intrafilas de los excombatientes, a excepción de la subcategoría cocina, dentro del campo trabajos técnicos, donde cuatro personas mencionaron el curso de panadería dado por el SENA en la ZVTN durante su proceso de reincorporación. Algo similar ocurre con la subcategoría construcción, en la que se agruparon ocho personas que hablaron de las nuevas habilidades o retos asumidos en la construcción de las viviendas en esta zona.

Tabla 1. Clasificación de las respuestas a las preguntas 1 y 6 de las cartillas

\begin{tabular}{|l|l|l|}
\hline $\begin{array}{c}\text { Campos de } \\
\text { aprendizaje }\end{array}$ & \begin{tabular}{c}
\multicolumn{1}{c|}{$\begin{array}{c}\text { Procesos de aprendizajes y } \\
\text { contenidos especificos intrafilas }\end{array}$} \\
Enfermería (12) \\
Medicina (9) \\
Laboratorio clínico (1) \\
Primeros auxilios (2) \\
Odontología (4)
\end{tabular} & $\begin{array}{l}\text { "Me he preparado en una escuela bá- } \\
\text { sica de medicina: lo he logrado por la } \\
\text { necesidad en el transcurso de la lucha } \\
\text { de atender nuestros mismos compañe- } \\
\text { ros y civiles". Cartilla 100, respuesta a } \\
\text { la pregunta 6. }\end{array}$ \\
\hline \multirow{5}{*}{ Educación/ } & $\begin{array}{l}\text { Educadores/pedagogía (7) } \\
\text { Aprender para enseñar la realidad del } \\
\text { país (40) } \\
\text { A ser educados (4) } \\
\text { Cultural (2) } \\
\text { Estudiar (6) } \\
\text { Trabajo organizativo (9) }\end{array}$ & $\begin{array}{l}\text { "Educar a las otras personas en cono- } \\
\text { cimientos y sabiduría, porque no hay } \\
\text { revolución sin educación". Cartilla 131, } \\
\text { respuesta a la pregunta 1. }\end{array}$ \\
\hline & $\begin{array}{l}\text { Emisora/locución (4) } \\
\text { Comunicaciones (8) } \\
\text { Radista (12) } \\
\text { Propaganda (3) } \\
\text { Sistemas (7) }\end{array}$ & $\begin{array}{l}\text { "Interactuar con las personas, me he } \\
\text { desempeñado como locutora, radista, } \\
\text { lo he logrado con sacrificio y la necesi- } \\
\text { dad de los trabajos en la organización". } \\
\text { Cartilla 110, respuesta a la pregunta 6. }\end{array}$ \\
\hline Comunicación (34)
\end{tabular}
parte de mi tesis doctoral en antropología. 


\begin{tabular}{|c|c|c|}
\hline $\begin{array}{l}\text { Campos de } \\
\text { aprendizaje }\end{array}$ & $\begin{array}{c}\text { Procesos de aprendizajes y } \\
\text { contenidos especificos intrafilas }\end{array}$ & Ejemplos de respuesta \\
\hline Valores (21) & $\begin{array}{l}\text { Solidaridad (3) } \\
\text { Respeto (6) } \\
\text { Responsabilidad (3) } \\
\text { Disciplina (4) } \\
\text { Conciencia (2) } \\
\text { Humildad (1) } \\
\text { Honestidad (1) } \\
\text { Esfuerzo (1) }\end{array}$ & $\begin{array}{l}\text { "He aprendido a trabajar en equipo y } \\
\text { siempre pensando en el colectivismo y } \\
\text { eliminando todos los rasgos pequeño- } \\
\text { burgueses de la sociedad capitalista. } \\
\text { Viendo todas las realidades que se ven } \\
\text { en el mundo contemporáneo". Cartilla } \\
98, \text { respuesta a la pregunta } 6 \text {. }\end{array}$ \\
\hline $\begin{array}{l}\text { Trabajos } \\
\text { "técnicos"17 (60) }\end{array}$ & $\begin{array}{l}\text { Construcción (17) } \\
\text { Cocina (14) } \\
\text { Panadería (4) } \\
\text { Manejo motosierra (2) } \\
\text { Agricultura (3) } \\
\text { Costura (4) } \\
\text { Conductor/mecánico (13) } \\
\text { Arte/artesanía (3) }\end{array}$ & $\begin{array}{l}\text { "Sastrería, nos llevaron a un campa- } \\
\text { mento donde se hacía y se practicaba } \\
\text { en retazos. A aprender también cocina, } \\
\text { con un compañero que tenía más } \\
\text { experiencia". Cartilla 141, respuesta a } \\
\text { la pregunta } 6 .\end{array}$ \\
\hline Alfabetización (15) & $\begin{array}{l}\text { Leer y escribir (5) } \\
\text { Leer (4) } \\
\text { Comprensión de lectura (3) } \\
\text { Escribir (3) }\end{array}$ & $\begin{array}{l}\text { "Leer y entender un texto. Lo he } \\
\text { logrado preguntando y desarrollando } \\
\text { actividades". Cartilla 27, respuesta a la } \\
\text { pregunta } 6 .\end{array}$ \\
\hline $\begin{array}{l}\text { Alfabetización } \\
\text { política }(34)^{18}\end{array}$ & $\begin{array}{l}\text { Político e ideológico (19) } \\
\text { Derechos (1) } \\
\text { Marxismo (4) } \\
\text { Lucha de clases (6) } \\
\text { Historia y realidad de Colombia (4) }\end{array}$ & $\begin{array}{l}\text { "Aprendizaje político y que todos } \\
\text { seamos unos guerrilleros integrales". } \\
\text { Cartilla 48, respuesta a la pregunta } 6 \text {. }\end{array}$ \\
\hline
\end{tabular}

17 Vale aclarar que algunos de los contenidos específicos mencionados en este recuadro son aprendizajes que los excombatientes han recibido en las ZVTN (año 2017), durante sus procesos de tránsito hacia la vida civil. Centros educativos como el SENA y la Universidad Industrial de Santander (UIS) han ofrecido talleres de panadería, cocina, etc.

Esta categoría se explora aquí en el sentido que le dio de Paulo Freire (1970), es decir, el sustantivo alfabetización aparece metafóricamente para explicar los procesos y retos de la alfabetización en personas adultas desde el punto de vista lingüístico. Esos procesos son considerados por este autor como esencialmente políticos. De esta reflexión emerge la noción de concientización, que se refiere al proceso por el cual los seres humanos se insertan críticamente en la acción transformadora de sus propias realidades. 


\begin{tabular}{|c|c|c|}
\hline $\begin{array}{l}\text { Campos de } \\
\text { aprendizaje }\end{array}$ & $\begin{array}{l}\text { Procesos de aprendizajes y } \\
\text { contenidos especificos intrafilas }\end{array}$ & Ejemplos de respuesta \\
\hline $\begin{array}{l}\text { Labores } \\
\text { administrativas (8) }\end{array}$ & $\begin{array}{l}\text { Administración (6) } \\
\text { Manejo de personal (2) }\end{array}$ & $\begin{array}{l}\text { "La principal es la conducción de } \\
\text { tropas y su preparación en las diversas } \\
\text { actividades. La he adquirido a través } \\
\text { de la educación y la práctica, lo que } \\
\text { nos ha permitido llegar con éxito al } \\
\text { momento actual". Cartilla 260, pre- } \\
\text { gunta 6. "Manejo los recursos para los } \\
\text { combatientes: intendencias, economía, } \\
\text { medicamentos, transporte, lo aprendí } \\
\text { en la práctica". Cartilla 105, respuesta } \\
\text { a la pregunta } 6 .\end{array}$ \\
\hline $\begin{array}{l}\text { Principios } \\
\text { revolucionarios (46) }\end{array}$ & $\begin{array}{l}\text { Vivir en comunidad / colectivismo (6) } \\
\text { Ser integral (5) } \\
\text { Trabajo (7) } \\
\text { Trabajar en unidad/conjunto (9) } \\
\text { Empatía (5) } \\
\text { Convivir con el medio ambiente (2) } \\
\text { Se aprenden muchas cosas (8) } \\
\text { Se aprende de todo un poco (3) } \\
\text { Dar la vida por la revolución (1) }\end{array}$ & $\begin{array}{l}\text { "Mejorar en todo aspecto para erra- } \\
\text { dicar el individualismo, la ignorancia, } \\
\text { la desigualdad y que sobresalga la } \\
\text { solidaridad y la tolerancia; sabiduría y } \\
\text { respeto por los demás, el colectivismo, } \\
\text { para expresarnos". Cartilla 56, respues- } \\
\text { ta a la pregunta } 1 .\end{array}$ \\
\hline $\begin{array}{l}\text { No sabe / no ha } \\
\text { tenido (2) }\end{array}$ & No sé (1), no he tenido (1) & $\begin{array}{l}\text { "No he tenido". Cartilla 148, respuesta } \\
\text { a la pregunta } 6 .\end{array}$ \\
\hline Otros (5) & $\begin{array}{l}\text { Ser alguien en la vida (1) } \\
\text { Ser activa (1) } \\
\text { Escuchar y ser escuchada (1) } \\
\text { Aprovechar el tiempo (1) } \\
\text { Alta autoestima (1) }\end{array}$ & $\begin{array}{l}\text { "He aprendido a escuchar y ser } \\
\text { escuchada". Cartilla 254, respuesta a la } \\
\text { pregunta } 6 .\end{array}$ \\
\hline
\end{tabular}

Fuente: elaboración propia.

Los contenidos específicos de la columna campos de aprendizaje se relacionan directamente con el eje argumentativo de mi propuesta sobre una pedagogía desde la paz, en cuanto procesos específicos de aprendizaje que ensamblan universos de memorias y conocimientos locales.

Se destacan las 68 menciones de los participantes agrupadas en el campo de aprendizaje “educación/pedagogía”, del cual 43 respuestas remiten al "enseñar la realidad de Colombia”, que es la respuesta más común. En una de las cartillas, un participante lo describe de la siguiente forma:

Las FARC es una escuela de aprendizaje, de enseñanza, de sabiduría, pertenencia al mundo real (las realidades del campo), allí se aprende a 
trabajar en colectivo, se erradica el individualismo, es decir, las FARC es la escuela donde se aprende la verdad. La importancia de que vivamos en una sociedad donde no tenemos discriminación ninguna e intercambiemos saberes entre muchos de nosotros. (Cartilla 227)

De aquí se deduce, en primer lugar, el interés de los excombatientes en reivindicarse como capaces de enseñar esas "realidades del país”, en gran medida porque este aprendizaje se recibió dentro de la “organización”. Sobre este saber, un análisis preliminar de las cartillas indica que tienen que ver con los siguientes presupuestos: 1) "no confiar en el Estado" (23 menciones), 2) “crítica a la "burguesía” (34 menciones), 3) “el interés del pueblo prima sobre el de los individuos” (28 menciones), 4) "la vida en el campo es el mundo real que el Estado ignora” (25 menciones). Se hace evidente una alta valoración de enseñar y aprender desde el trabajo colectivo. Estas ideas, además, aparecen de manera recurrente en las memorias y narrativas recogidas en las cartillas, tanto en la categoría sobre pedagogías (40 menciones), como en las de “alfabetización política” (4) y “principios revolucionarios" (con 6 menciones en el subcampo de vivir en comunidad y 9 para el de trabajo en unidad).

Dentro de sus respuestas, las "realidades del país" —o sea las "realidades del campo" y de la guerra- a su vez se encuentran hiladas a una narrativa sobre los principios revolucionarios, otra categoría émica sobresaliente en los resultados (con 45 menciones), así como con sus expectativas para el futuro (figuras 3 y 4).

El cruce entre el campo de aprendizaje categorizado como pedagogía con el de principios revolucionarios indica que para estos excombatientes la pedagogía y la educación en la transición hacia la vida civil dentro del proceso de paz están relacionadas con enseñar la realidad del campo y de la guerra a través de una reelaboración de los principios revolucionarios, expresados en sus memorias de la siguiente manera (tabla 1): vivir en comunidad/colectivismo (6), ser integral (5), trabajo (7), trabajar en unidad/conjunto (9), empatía (5), convivir con el medio ambiente (2), dar la vida por la revolución (1). Hay entonces una pedagogía que se expresa y se enseña desde memorias de una vida de resistencia en un mundo considerado real, rural y "en la guerra”, como lo sugieren respuestas a la pregunta 6 sobre la importancia de lo aprendido en las escuelas de formación de las FARC-EP para su incorporación a la vida civil:

Sí tiene importancia [la educación dentro de las FARC-EP] porque muchos no teníamos esa educación en el campo, cuando seamos civiles no nos vamos a quedar con lo que dicen los libros sino mirar las realidades. Es importante para no dejarnos enredar, sino analizar lo que ocurre en el país. (Cartilla 52, participante anónimo) 
Y en la cartilla 116: "Demasiada importancia, porque aprendimos muchas cosas que los civiles ni saben. Por ejemplo, no saben por qué existió las FARC, solo nos tildan de terroristas” (participante anónimo).

La alusión recurrente en estas cartillas a un conocimiento local que revela una "realidad del campo" que debe ser enseñada a todos los "civiles" es un dato que no debe tomarse a la ligera porque tiene un valor significativo para una pedagogía desde la paz; debería pensarse como un aspecto relevante dentro de los proyectos para la incorporación a la vida civil de los excombatientes. Ser un "profesional revolucionario" (figura 4) también tiene que ver con esta reflexión, es decir, con replanteamientos a partir de propuestas educativas desde las memorias y los procesos internos de aprendizaje en el marco de una pedagogía desde la paz.

Figura 3. Respuesta a la pregunta 4 sobre los lugares donde estudiaron antes de pertenecer a la organización

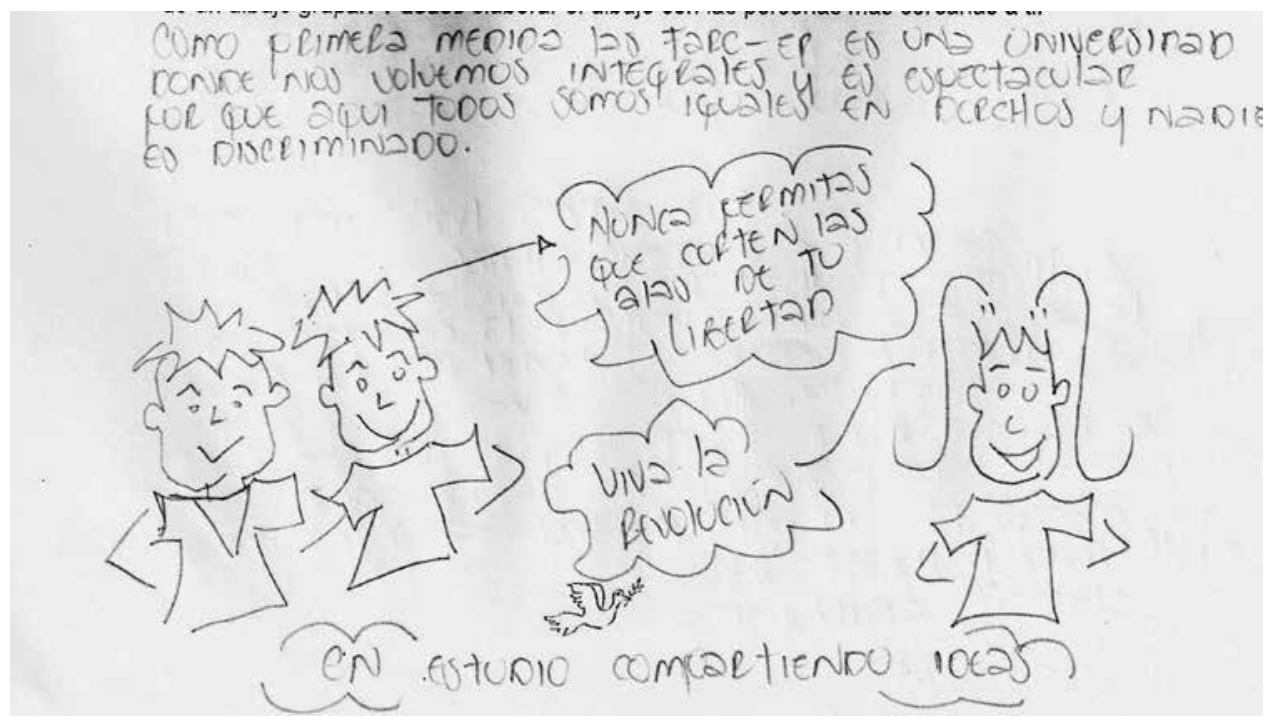

Fuente: fotografia de la autora, taller 4. 
Figura 4. Respuesta a la pregunta 5 sobre la importancia de las escuelas de formación de las FARC-EP

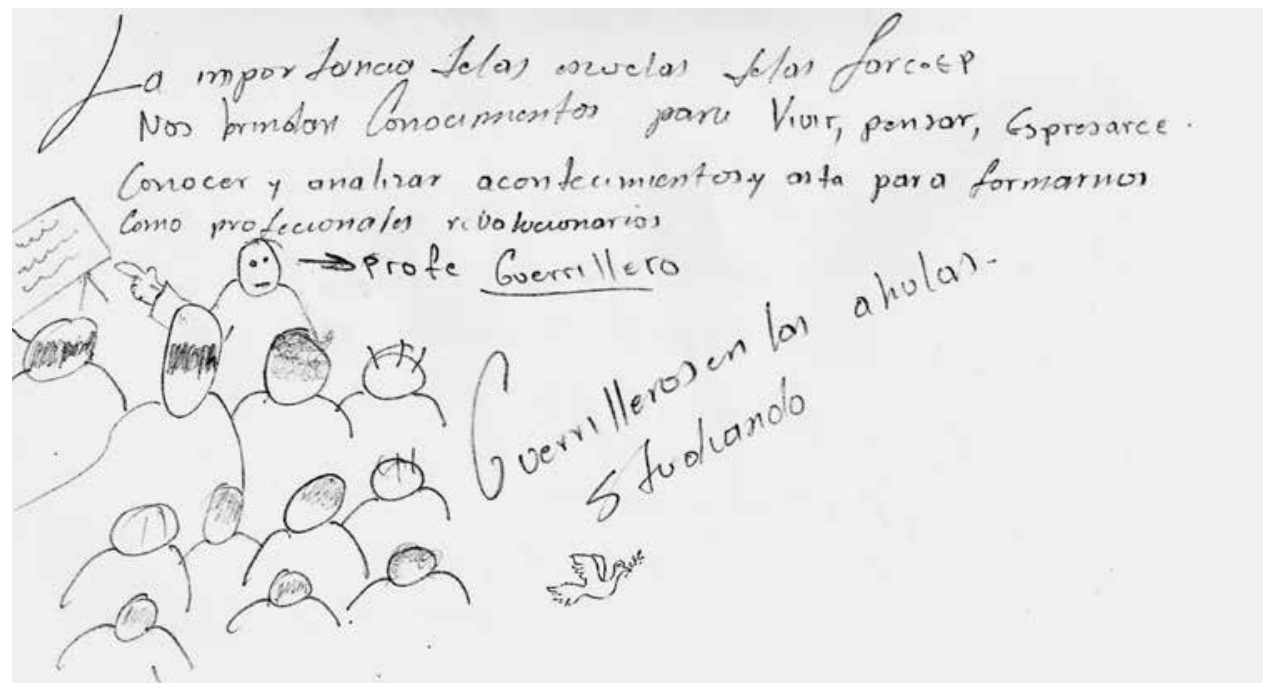

Fuente: fotografía de la autora, taller 4.

Las tendencias que arrojan algunos de los cruces preliminares entre campos y subcampos, así como las memorias contenidas en las ilustraciones, cumplen con un doble objetivo con relación al propósito de mostrar el valor de los procesos educativos locales de los excombatientes durante la etapa de su reincorporación a la vida civil. En primera instancia, permiten reconocer las memorias como lugares de disputas, conflictos y luchas, pero principalmente como procesos educativos, lo cual apunta a:

Prestar atención al rol activo y productor de sentido de los participantes en esas luchas, enmarcados en relaciones de poder. A "historizar" las memorias, o sea, reconocer que existen cambios históricos en el sentido del pasado, así como en el lugar asignado a las memorias en diferentes sociedades, climas culturales, espacios de luchas políticas e ideológicas. (Jelin 2002, 28)

Una vía para analizar el papel activo y a la vez histórico de los excombatientes dentro del proceso de paz vigente en Colombia es trabajar junto con y desde sus memorias sobre el conflicto. Esto supone ampliar los marcos ideológicos y conceptuales sobre los cuales se está edificando la paz, incluyendo miradas que denotan otros principios, valores y procesos.

Las subcategorías en los campos de educación/pedagogía y alfabetización política presentes en la tabla de resultados ponen de relieve, en segunda 
instancia, procesos de aprendizaje en los que el lugar que se le asigna a la memoria cumple su propósito como mecanismo de resistencia y lucha con miras al futuro; sus historias sobre cómo han aprendido (en la guerrilla) a "vivir en comunidad”, "trabajar en unidad”, “tener empatía por el otro" o "a enseñar la realidad del país” se convierten, dentro de un escenario de posconflicto, en memorias útiles para la transición.

\section{Consideraciones finales}

Los resultados que arrojan las cartillas contribuyen a una pedagogía desde la paz. Estos develan dos ejes centrales que pueden ser parte de un futuro proyecto educativo en el marco del posconflicto: el primero es el reconocimiento de las realidades locales que los excombatientes están dispuestos a compartir en su vida como civiles, pues, dentro de la guerrilla, fueron formados en lo que consideran "realidades de la guerra y del campo" y que, en el contexto de transición, se expresan mediante la renuncia -y reelaboración- de los principios revolucionarios (tabla 1). Segundo, es necesario ahondar en las expectativas de futuro de esta población porque en ellas filtran elementos de esos conocimientos locales, que son aportes centrales al proceso de paz, pues integran datos concretos para proyectos de inclusión.

La mayoría de las cartillas trabajadas con los excombatientes presentan diversas memorias y anhelos frente a su pasado guerrillero, su presente como excombatientes y su futuro como ciudadanos. También expresan qué significó vivir ese momento único e histórico de transición: el traslado a las ZVTN. Esta etapa de cambio fue ideal para explorar sus memorias del conflicto por medio de los talleres, pues contamos con la posibilidad de entrar a un mundo de expectativas diversas muy vivas. De hecho, el ejercicio pone de presente la urgencia de un diálogo con las miradas estatales oficiales del conflicto armado que son presentadas como coherentes, pero que necesitan ser problematizadas. La multiplicidad de relatos y dibujos encontrados en las cartillas sobre el camino de algunas mujeres para convertirse en enfermeras, las historias de las personas que aprendieron de radio o comunicación, las memorias de los exguerrilleros sobre cómo aprendieron a leer y escribir en medio del conflicto armado reflejan un deseo de trascender la lógica de la guerra y continuar un proceso formativo dentro de los campos de aprendizaje que empezaron a explorar cuando estaban en la organización guerrillera. También dejan en evidencia que la educación es 
un dominio que excede el campo de la educación formal, y que opera dentro de la cultura como un mecanismo de identidad, resistencia y lucha. Por ello, la propuesta de hacer pedagogía desde la paz cobra relevancia, en la medida en que interpela el campo de la educación formal, les da valor a los conocimientos locales y refuerza su inclusión en un escenario nacional de posconflicto.

La exploración colectiva de la memoria se convierte en un elemento esencial para la transformación de las condiciones de existencia y aspiraciones de un colectivo. El ejercicio presentado en este artículo da cuenta de una lucha política por la inclusión y el reconocimiento de dinámicas educativas locales durante la fase de implementación de los acuerdos de paz; una disputa que además se dirige contra el olvido de una guerra que marcó por siempre los proyectos de vida de varias generaciones. Da cuenta, además, de la urgencia de incorporar sus prácticas educativas en escenarios de construcción de paz. Estas memorias de grupos, poblaciones y comunidades que han padecido la guerra, entre ellos, los excombatientes de la guerrilla, demandan la intersección con las prácticas educativas nacionales vigentes.

La atención a las miradas locales del conflicto debe ser una prioridad dentro del proceso de paz en Colombia; el registro de procesos de aprendizaje internos y locales, así como la exploración de memorias y narrativas de comunidades que vivieron el conflicto desde adentro, puede contribuir a un proyecto incluyente orientado hacia una narrativa nacional de construcción desde la paz, una narrativa que desafíe aquella vieja alusión de bandos, de aliados y enemigos. La inclusión pasa por reconocer las experiencias de aprendizaje durante la guerra; legitimarlas dentro de sociedades que se encuentran en tránsito hacia la superación de violencias y en el camino hacia la construcción de paz desde las regiones. La inclusión significa reconocer que los excombatientes tienen unos deseos y narrativas sobre cómo imaginan su futuro, así como su lugar en la sociedad. Implica entender que la normatividad desde la cual se piensan la paz, la reconciliación, la educación y el posconflicto en Colombia se encuentra mediada por valores, ambigüedades y moralidades, y que el camino para lidiar con tales percepciones es un terreno de tensiones, conflictos y disputas políticas.

La inclusión de memorias y procesos pedagógicos locales en la implementación de políticas regionales en el proceso de paz es un asunto que merece ser debatido en varias disciplinas académicas, incluyendo la antropología, pues ese terreno de conflictos y luchas con el Estado a su vez pasa por problematizar "los modos concretos por los cuales los individuos y grupos sociales son tratados, según cuáles principios y en nombre de qué moral" (Fassin 2009, 57). El Estado colombiano debe escuchar a los excombatientes y abrir canales para un diálogo 
efectivo desde una escucha real de las memorias del conflicto y desde los procesos de aprendizaje locales; allí también hay múltiples posibilidades para construir la paz.

Esta no es una tarea fácil porque requiere problematizar las memorias hegemónicas así como sus sitios tradicionales de enunciación: el Estado y la escuela. Urge tender un puente entre estas miradas oficiales y las prácticas educativas locales, especialmente en épocas de recrudecimiento de la violencia sociopolítica, con los asesinatos de personas que lideran muchos de esos procesos en lo local. Los desafíos que enfrentan los procesos pedagógicos y educativos en el marco del posconflicto son enormes, pero las perspectivas de trabajo desde las comunidades locales son tan ricas y esperanzadoras como para poder enfrentarlos.

\section{Referencias}

Ávila, Rafael. 1994. La educación y el proyecto de la modernidad. Bogotá: Ediciones Antropos.

Bastide, Roger. 1983. “A propósito da poesia como método sociológico: textos de Roger Bastide”. Consultado el 25 de septiembre del 2019. https://projetobrasilfranca.wordpress. com/2010/07/12/a-proposito-da-poesia-como-metodo-sociologico-textos-de-roger-bastide/

Caicedo, Alhena y Carlos Alberto Benavides Mora. 2017. "La ZVTN de Buenos Aires, Cauca: un enfoque de paz desde los territorios”. Ponencia presentada en el XVI Congreso de Antropología en Colombia, Pontificia Universidad Javeriana, Bogotá, 6-9 de junio.

Fassin, Didier. 2009. “Another Politics of Life is Possible”. Theory, Culture \& Society 26 (5): 44-60. https://doi.org/10.1177/0263276409106349

Fernandes, Florestan y Ramzia Gattas. 1971. "A história de vida na investigação sociológica: a seleção de sujeitos e suas implicações”. En Ensaios de sociologia geral e aplicada, editado por Florestan Fernandes, 251-269. São Paulo: Livraria Pioneira Editora.

Freire, Paulo. 1970. El proceso de alfabetización política: una introducción. Ginebra: Institute of Cultural Action.

García Figueroa, Vianney Judith y Libia Tattay. 2017. “Tensiones y desafíos de las zonas veredales de normalización en territorios indígenas: una reflexión desde el corazón del pueblo nasa en el departamento del Cauca”. Ponencia presentada en el XVI Congreso de Antropología en Colombia, Pontificia Universidad Javeriana, Bogotá, 6-9 de junio.

“Glosario Icfes”. 2019. Consultado el 25 de septiembre del 2019. https://www.icfes.gov.co/ glosario

Hoyos G., Juan Felipe. 2017. "Territorios entretejidos: la zona de normalización de la Playa, Tumaco, entre la trama de violencia regional y el horizonte de transición”. Ponencia pre- 
sentada en el XVI Congreso de Antropología en Colombia, Pontificia Universidad Javeriana, Bogotá, 6-9 de junio.

Jelin, Elizabeth. 2002. Los trabajos de la memoria. Madrid: Siglo XXI.

Marín González, Keren y Nicolás Espinosa Espinosa. 2017. "Normalización sin transición: la dimensión territorial del proceso de paz en la zona veredal de transición y normalización (ZVTN) de La Macarena”. Agora USB 17 (2): 441-461. http://dx.doi. org/10.21500/16578031.3281

Oficina del Alto Comisionado para la Paz. 2017. "Funcionamiento de las zonas veredales transitorias de normalización (ZVTN) y los puntos transitorios de normalización (PTN)". Consultado el 25 de septiembre del 2019. http://www.altocomisionadoparalapaz.gov.co/ procesos-y-conversaciones/Documentos\%20compartidos/ABC-Zonas-veredales.pdf

Pollak, Michael. 1989. "Memória, esquecimento, silêncio”. Revista Estudos Históricos 2 (3): 3-15. http://www.uel.br/cch/cdph/arqtxt/Memoria_esquecimento_silencio.pdf

Queiroz, Maria Isaura Pereira de. 1988. Variações sobre a técnica de gravador no registro da informação viva, vol. 4. São Paulo: Universidade de São Paulo.

“Sembrando oportunidades. Informe de gestión 2016-2017”. 2017. Educapaz. https://educapaz.co/leenos/informes-de-gestion/

Valero, Rodrigo. 2017. “Desde las veredas vecinas: la ZVTN el Oso en Planadas, Tolima”. Ponencia presentada en el XVI Congreso Colombiano de Antropología, Pontificia Universidad Javeriana, Bogotá, 6-9 de junio.

Veeduría Ciudadana Campamento por la Paz. 2016. Veeduría Ciudadana Campamento por la Paz, PDCPL 21-481 Personería de Bogotá. Bogotá: Veeduría Ciudadana Campamento por la Paz.

Villa, Juan David. 2009. "La memoria como territorio en disputa y fuente de poder: un camino hacia la dignificación de las víctimas y la resistencia no violenta”. En Recordar en conflicto: iniciativas no oficiales de memoria en Colombia. Bogotá: Centro Internacional para la Justicia Transicional (ICTJ).

Walsh, Catherine E. 2013. Pedagogías decoloniales: prácticas insurgentes de resistir, (re)existiry (re)vivir. Quito: Abya Yala.

Zuluaga, Olga, Alberto Echeverri, Alberto Martínez, Stella Restrepo y Humberto Quiceno. 1988. "Educación y pedagogía: una diferencia necesaria”. Revista Educación y Cultura 14: 4-9. http://www.albertomartinezboom.com/escritos/articulos/2013_Educacion_y_pedagogia_Una_diferencia_necesaria.pdf 